\title{
Blood Particle Trajectories in Phase-Contrast-MRI as Minimal Paths Computed with Anisotropic Fast Marching
}

\author{
Michael Schwenke ${ }^{1}$, Anja Hennemuth ${ }^{1}$, Bernd Fischer ${ }^{2}$, Ola Friman ${ }^{1}$ \\ ${ }^{1}$ Fraunhofer MEVIS, Institute for Medical Image Computing, Bremen \\ ${ }^{2}$ Institute of Mathematics and Image Computing, Universität zu Lübeck \\ michael.schwenke@mevis.fraunhofer.de
}

\begin{abstract}
In this paper the well-established minimal path framework is applied to the problem of computing blood flow trajectories in phase contrast magnetic resonance imaging (PC-MRI). The velocity vectors measured by PC-MRI and an uncertainty measure are combined to a metric tensor and minimal paths are calculated with anisotropic fast marching method (FMM). Exemplary results of the computation of the blood particle trajectories are given. This work contributes a novel application of anisotropic FMM to flow computations.
\end{abstract}

\section{Introduction}

Phase constrast magnetic resonance imaging (PC-MRI) is a noninvasive technique capable of accurately measuring $3 \mathrm{D}+\mathrm{t}$ flow velocities. It has a variety of established applications in quantifying cardiovascular function and hemodynamics [1]. Traditional visualization techniques, such as vector glyphs, streamlines, pathlines and particle traces are frequently employed for visualizing blood flow based on the velocity data [2]. Clinical applications that benefit from such flow pattern information include the assessment of stenoses, aneurysms, and heart valve function, the development of vessel plaque and surgical planning and follow-up in congenital heart disease [2]. Whereas the traditional method for computing flow trajectories is a deterministic streamlining approach, working solely on the given velocity vectors, the authors of [2] derive the statistical properties of the PC-MR images and incorporate these properties into the tracking of particle trajectories. Using a sequential Monte Carlo method, a blood flow mapping is computed giving information on the likelihood of the trajectory taking a certain path through the velocity vector field.

In this work, an alternative approach to the computation of particle trajectories is explored in the framework of minimal paths in anisotropic media.

\section{Materials and Methods}

\subsection{Minimal Paths in Anisotropic Media}

A minimal path in a medium is a path which minimizes a certain metric along the path, e.g. a ray of light takes the min-time path through an optical medium. 
In the case of an isotropic local metric, which does not depend on the direction of motion, one can compute minimal paths with the standard FMM. In case of anisotropic local metric, i.e. the metric depends on the direction of motion, the traditional FMM cannot be applied directly. We here focus on anisotropic media with locally ellipsoidal shape. Formally, let $\Gamma_{x, \partial \Omega}$ be the set of all possible paths $\gamma: \mathbb{R}_{0,+} \rightarrow \Omega$ connecting a point $x \in \Omega$ to a given point of interest $y \in \partial \Omega$. Further let

$$
F\left(s, \gamma, \gamma^{\prime}\right)=\sqrt{\gamma^{\prime}(s)^{T} D_{\gamma(s)}^{-1} \gamma^{\prime}(s)}
$$

measure an infinitesimal distance along a path $\gamma$ relative to the inverse of a symmetric positive definite metric tensor $D$. In an isotropic setting, $D=f(x) I$ and this equation reduces to the Eikonal equation known from standard FMM. Then a minimal path is a path $\gamma$ minimizing the functional

$$
J(\gamma)=\int_{\gamma} F\left(s, \gamma, \gamma^{\prime}\right) \mathrm{d} s
$$

Following the dynamic programming approach, minimal paths from all points $x \in \Omega$ to the target are computed simultaneously. To this end, the so-called value function is defined

$$
\begin{array}{lll}
U(x)=\min _{\gamma \in \Gamma_{x, \partial \Omega}} J(\gamma)=\min _{\gamma \in \Gamma_{x, \partial \Omega} \int_{\gamma} F\left(s, \gamma, \gamma^{\prime}\right) \mathrm{d} s,} & \forall x \in \Omega \\
U(x)=q(x), & & \forall x \in \partial \Omega
\end{array}
$$

The value function $U$ satisfies the static Hamilton-Jacobi equation

$$
\forall x \in \Omega, \quad \nabla U(x)^{T} D_{x} \nabla U(x)=1
$$

and the tangents of the minimal paths satisfy $\gamma^{\prime} \propto D \nabla U$, see [3] for proofs of both properties. Thus, we are able to solve the problem of computing the minimal paths by first solving the boundary problem starting from a given value $q(x)$ on the start point $x \in \partial \Omega$ (the particle of interest) and reconstruct the minimal paths afterwards by solving the ordinary differential equation $\gamma^{\prime}(s) \propto$ $-D_{\gamma(s)} \nabla U(\gamma(s))$, with $\gamma(0)=x$ using standard numerical methods like Heun's or Runge-Kutta's.

\subsection{Anisotropic Fast Marching Method}

To compute the solution of the PDE in ((4)) anisotropic FMM is used. A detailed description of the numerical details is unfortunately not possible due to space limitations. The main propositions in literature for anisotropic FMM are the ordered-upwind methods [4], a derivation of these [3], a control-theoretic approach [5] and a recursive correction FMM [6]. Based on numerical tests we find a combination of [3] and [6] to be most suitable for the anisotropies we are faced with. We use 26-neighbors in the local update computation, which gives accurate results up to relatively high anisotropies, as shown in [3] and as we have confirmed with our implementation. Contrary to [3], we only use direct neighbors 
for the propagation, which greatly speeds up computation. Furthermore, in contrast to the standard FMM, we also utilize TRIAL points, for which only an approximation to the correct value of $U$ exists, in the computation of $U$ resulting in a higher accuracy of the standard FMM solver. We combine this scheme with the method of [6] which provides a recursive correction for the errors introduced by the FMM solver in anisotropic media. Locally, we have to solve a constrained optimization problem, which in case of the elliptical speed profile has an analytical solution as shown in [3].

\subsection{Connectivity Measure}

The FMM connects every point of the domain to the source point by a minimal path. In this section the focus is on how to differentiate more likely paths from less likely paths. Let $\mathcal{C}(x)=\sqrt{\gamma^{\prime}(x)^{T} D_{x} \gamma^{\prime}(x)}$ be a local measure describing the alignment of the minimal path starting at $x$ with the major eigenvector of the metric tensor $D$. This metric gives a high value if $\gamma^{\prime}(x)$ and the major eigenvector are aligned, whereas it gives a low value if $\gamma^{\prime}(x)$ and the smallest eigenvector are aligned. The mean value of $\mathcal{C}$ along the path is high for more likely paths. This kind of connectivity measure was proposed in [5] in the context of fiber tracking based on Diffusion Tensor Imaging.

\subsection{Modeling Blood Particle Trajectories as Minimal Path}

The PC-MRI velocity vectors represents the flow at a position in $\mathrm{m} / \mathrm{s}$. This measure is subject to measurement errors for which the authors of [2] derive the statistical properties. We incorporate the uncertainty of the measure into a metric tensor and then solve the minimal path problem in this metric space. This allows the tangents of the paths to differ from the velocity vectors to a certain amount, controlled by the uncertainty. Let $v_{x}$ be the local blood flow velocity vector measured at position $x \in \Omega$ and $\sigma$ be the uncertainty of the measurement of the velocity vector. A metric tensor is then constructed as

$$
D_{x}=v_{x} v_{x}^{T}+\sigma I
$$

(Fig. 1). In the following, we take the $3 \mathrm{D}$ vector field to be constant over time, future work will include working on the full 3D-temporal PC-MR image sequence. We restrict the FM to only propagate along the range of direction of the velocity vector \pm 90 degrees.

\section{Results}

Evaluation was done on two PC-MRI data sets. One dataset is from a healthy subject (P1), one from a patient with an aneurysm (P2) disturbing blood flow. Both datasets are $3 \mathrm{D}+\mathrm{t}$ with 14 time steps reconstructed for one cardiac cycle. The ROI for $\mathrm{P} 1$ is of size $50 \times 160 \times 24$ and voxelsize is $1.67 \times 1.67 \times 3.5 \mathrm{~mm}^{3}$. 
The ROI for P2 is $68 \times 190 \times 28$ with voxelsize $1.77 \times 1.77 \times 2.6 \mathrm{~mm}^{3}$ and we compute the trajectories on one timepoint. In both dataset maximal velocity is $1500 \mathrm{~mm} / \mathrm{s}$ and we set the uncertainty to $\sigma=50 / 1500=0.033$, where $50 \mathrm{~mm} / \mathrm{s}$ is the variance of measurement derived in [2]. A user-given particle of interest, the direction of propagation (forward or backwards in time) and the maximal Euclidean length of the paths are required as input. Fig. 2 shows results of the computation of the particle trajectories for P1. Each row is giving the results for a different input. The trajectories are smooth with only small disturbances. Fig. 3 shows results of the computation of the particle trajectories for P2. Trajectories are computed for three different ranges of connectivity measure. Computation times vary from 5 to 20 seconds in the presented cases.

\section{Discussion}

In this paper we propose a modeling of blood particle trajectories as minimal paths through an anisotropic medium. Future work includes extension to 3D-

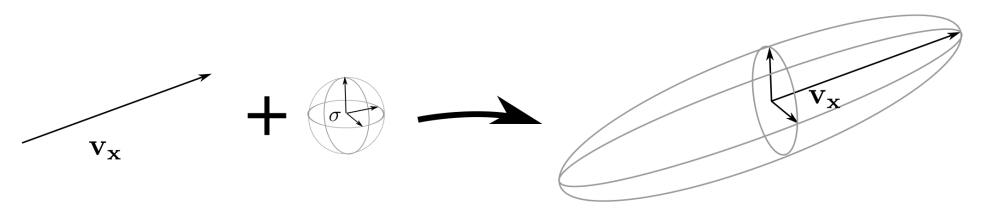

Fig. 1. Construction of the tensor from the given PC-MRI velocity vector.

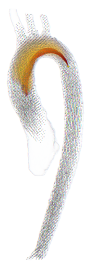

(a)

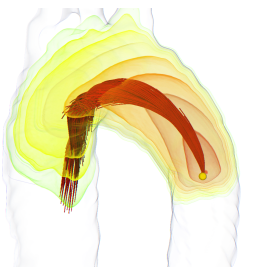

(b)

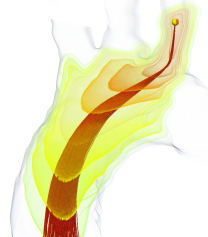

(e)
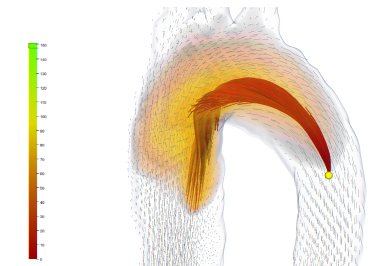

(c)
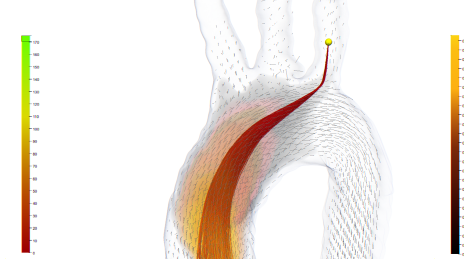

(f)

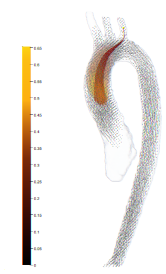

(d)

Fig. 2. Blood flow trajectories computed on data of a healthy subject. Each row shows a different particle of interest. Left: Aorta with superimposed PC-MRI velocity vectors, connectivity and trajectories. Middle: Close-up with geodesic distance map iso-volume rendering. Right: Close-up with PC-MRI-vectors, connectivity and paths. The paths are computed from the points with highest $10 \%$ of mean connectivity value. 
Fig. 3. Results for a patient with an aortic aneurysm. The trajectories are computed for different intervals of connectivity: (a) $90-100 \%$, (b) $60-61 \%$, (c) $50-51 \%$. Notice that the trajectories in (c) may transport plaque particles from the right wall of the aorta up to the left subclavian artery. This information is useful to rate the severity of plaque in different areas.

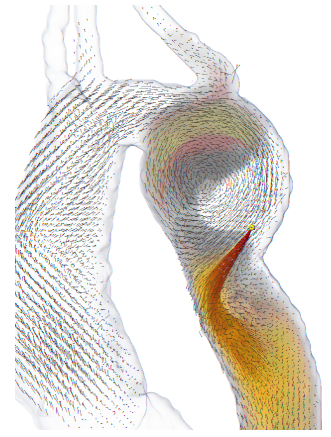

(a)

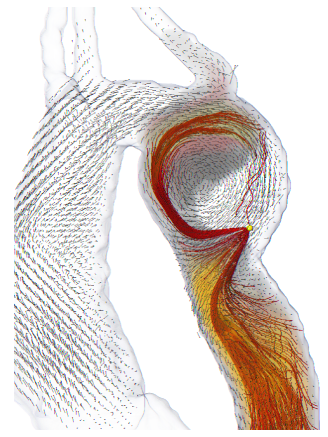

(b)

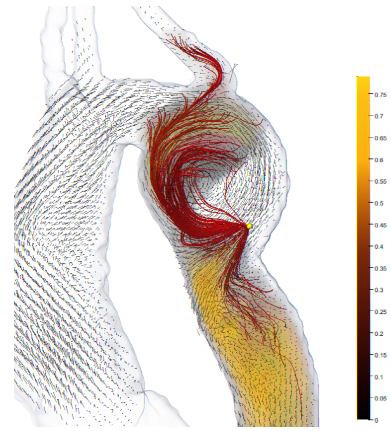

(c)

temporal image sequences (4D images) to allow for computation of swirls in the blood flow, where a trajectory may visit a 3D point several times. Furthermore we plan on working on physical evaluation and further evaluations on different data sets.

\section{Acknowledgements}

The authors would like to thank Dr. Michael Markl at Universitätsklinikum Freiburg for providing the data.

\section{References}

1. Srichai MB, Lim RP, Wong S, et al. Cardiovascular applications of phase-contrast MRI. Am J Roentgenol. 2009;192(3):662-75.

2. Friman O, Hennemuth A, Harloff A, et al. Probabilistic 4D blood flow mapping. Lect Notes Computer Sci. 2010;6363:416-23.

3. Jbabdi S, Bellec P, Toro R, et al. Accurate anisotropic fast marching for diffusionbased geodesic tractography. Int J Biomed Imaging. 2008;2008:2:1-2:12.

4. Sethian JA, Vladimirsky A. Ordered upwind methods for static Hamilton-Jacobi equations: theory and algorithms. SIAM J Numer Anal. 2003;41(1):325-63.

5. Prados E, Lenglet C, Pons JP, et al. Control theory and fast marching techniques for brain connectivity mapping. Proc CVPR. 2006; p. 1076-83.

6. Konukoglu E, Sermesant M, Clatz O, et al. A recursive anisotropic fast marching approach to reaction diffusion. Proc IPMI. 2007; p. 687-99. 